\title{
Use of high resolution SAR data for the monitoring of water resources in Burkina Faso
}

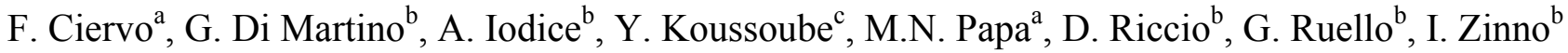 \\ ${ }^{a}$ Università di Salerno, Dipartimento di Ingegneria Civile, Italy; \\ ${ }^{\mathrm{b}}$ Università di Napoli "Federico II", DIBET, Via Claudio 21, 80125, Napoli, Italy \\ ${ }^{\mathrm{c}}$ Departement de Geologie, UFR/SVT - University of Ouagadougou, Burkina Faso
}

\begin{abstract}
The integrated management of water resources is a crucial problem for improving the quality of life in Sub-Saharian Africa. Several satellites everyday acquire a huge amount of physical information that could be employed as a support for solving agriculture and water problems. In this paper we present a project devoted to exploit the use of high resolution synthetic aperture radar (SAR) images for water resource management at no cost for the users. A case study is developed in the Yatenga region, in the northern Burkina Faso, integrating hydrologic and remote sensing models in order to improve the capacity of predicting flood and drought events. Main attention is posed here on the innovative fractal techniques developed for the extraction of geometrical and physical parameters that can be used for calibrating hydro-geological models.
\end{abstract}

Keywords: Synthetic Aperture Radar, developing countries, water management.

\section{INTRODUCTION}

Burkina Faso is a West Africa country, located in the semi-arid area between the Sahara desert and the gulf of Guinea. The country is characterized by a Sahelian climate, with the alternation of rainy ( 3 months) and dry ( 9 months) seasons. In this context, an efficient water resource management is a key goal for improving the people quality of life. Recently, several remote sensing sensors were launched and they acquire a huge amount of physical information in every corner of the Earth. In this paper we present a research project whose goal is the innovative use of remotely sensed data for water monitoring in semi-arid regions. In particular, the project is developed on the northern area of Burkina Faso, in the Yatenga region. The use of remotely sensed data gives a synoptic view of wide areas, and it provides a support for several activities related with the water management, as the identification, storage and distribution of the resource. Therefore, we propose to integrate hydrological and remote sensing models and GIS techniques for water resource monitoring. In particular, we use Cosmo-SkyMed data obtained in the frame of a research project approved by the Italian Space Agency (ASI). A set of strip-map, spotlight and scan-sar data is provided from April 2010 to March 2012.

Section 2 of the paper will be devoted to present the study area and the main characterics of the available COSMOSkyMed images.

In Section 3 we present the project rationale, with particular attention posed on the hydrological and remote sensing models that we are developing for the project. The proposed models will be integrated with the aid of Geographic Information Systems (GIS).

In this paper, main emphasis will be posed on the presentation of innovative techniques devoted to estimate the fractal parameters of the observed area. Such a goal is particularly important because the fractal parameters are related to the surface roughness, whose value can be indicative of phenomena that are related to natural events. For instance, the identification of flooded areas that are very common during the rainy season can be performed by comparison of fractal parameters value of pre- and post-event images [1]. Direct and inverse models were developed and their application to this case study will be discussed. In particular, for surface description purposes, we use an fBm model, a regular stochastic model defined in terms of only two parameters. Such an easy and reliable model allows the expression of the surface spectrum by means of a linear relation in a log-log plane. A relation between image and surface spectrum is also found in order to extract the surface fractal parameters from the SAR images. The details of the technique are presented in Section 4, where some details will be provided on the extraction of the Digital Elevation Model (DEM) from the

SAR Image Analysis, Modeling, and Techniques XI,

edited by Claudia Notarnicola, Simonetta Paloscia, Nazzareno Pierdicca, Proc. of SPIE Vol. 8179, 817909

(C) 2011 SPIE · CCC code: 0277-786X/11/\$18 - doi: 10.1117/12.898339

Proc. of SPIE Vol. $8179817909-1$ 
images, needed for the hydro-morphological watershed characterization for the hydrological catchment response. The integration of data and results will be performed with the intense use of geographic information systems, that provide the production of value added maps that can be easily understood by non-expert users. A discussion on the project potentiality and on the future developments is provided in the conclusions section.

\section{THE YATENGA REGION SEEN BY COSMO-SKYMED}

\subsection{The Study Area}

Burkina Faso is a continental country located in West Africa more than $1000 \mathrm{~km}$ from the Gulf of Guinea. It has approximately 14 milion people, most of which lives in rural areas depending from agriculture and livestock [2]. The last years of the 20th century (from 1970 to 2000) were marked by a long period of drought. A return to normal rainfall rates is experienced since the beginning of the 2000s when the average rainfall ranges from $350 \mathrm{~mm}$ per year in the North to $1200 \mathrm{~mm}$ per year in the South of the country, according to a latitudinal gradient [3]. The annual rainfall is not uniformly distributed, with a rainy season from May to September and dry season from October to April. Rapid regime of rainy event causes a crusting of the soil and a significant runoff. The air temperature varies with a maximum monthly average of $41^{\circ} \mathrm{C}$ (March - April) and a minimum monthly average of $17^{\circ} \mathrm{C}$ (December - January) for the period 1965-2006. The evaporation is about $2000 \mathrm{~mm}$ per year so that at the end of the dry season only the surface water bodies with more than $3 \mathrm{~m}$ of height survive. The long drought period and some inadequate cultivation practices resulted in a degradation of land and a high runoff. The infiltration of water is so limited.

The SAR images cover an area of about $40 \mathrm{~km} \times 40 \mathrm{~km}$, near the city of Ouahigouya, capital of the Yatenga district, in the North of Burkina Faso. In Figure 1 we show an optical image of the area of interest, with a white frame that identifies the region illuminated by the SAR sensor. The hills that cross the area from the upper right corner to the city of Ouahigouya (point A) represent the watershed that separates the Black and the White Volta basins.

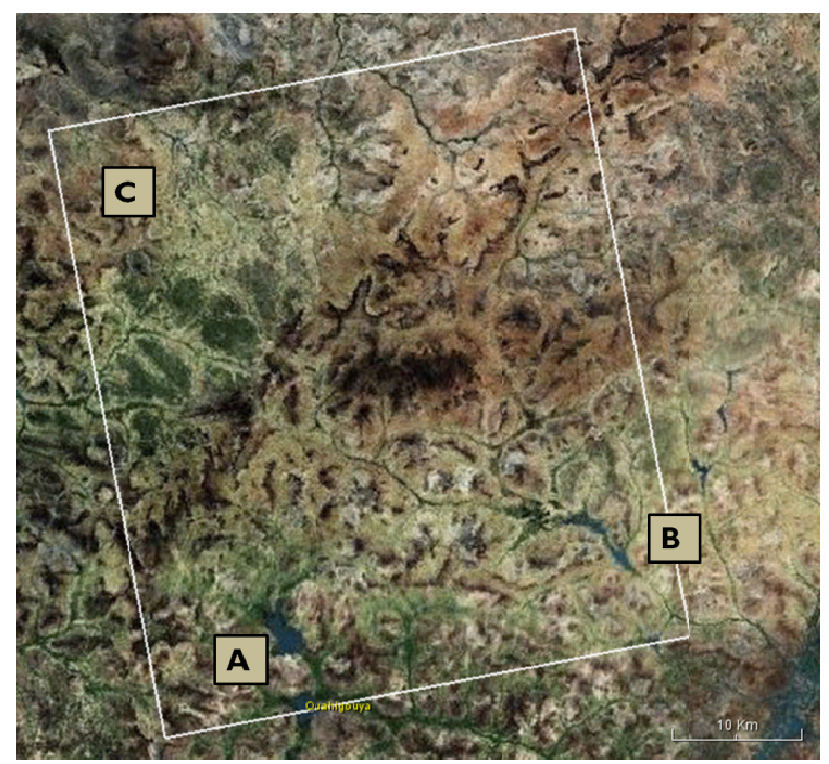

Figure 1: Synoptic view of the area of interest. The white frame identifies the imaged area

The boxes $\mathrm{B}$ and $\mathrm{C}$ in Figure 1 represent the location of the Tougou and Bidi basins, that are the sub-basins object of our studies. The area is almost flat, with heights ranging about from 300 to 400 meters. The scenery is strongly variable between the dry and the rainy season. SAR sensors are very sensitive to these changes, as shown in the following sections. 


\subsection{Cosmo-SkyMed SAR images}

Cosmo-SkyMed is a constellation of satellites that acquire X band SAR images with a resolution of the order of the meter or less. Thanks to the high sensitivity of SAR data to soil moisture and surface roughness, the Cosmo-SkyMed acquisitions can be very useful to the hydrological modeling, if appropriate interpretative models and reliable retrieval techniques are developed.

In Figure 2 two samples of the acquired data are shown. In particular, in Figure $2 \mathrm{a}$ and $2 \mathrm{~b}$ we present the data acquired on the 14 of July and the 15 of August 2010, respectively. The variation between the fields scattered with a temporal interval of one month is significant. This is due to the intense precipitations that fall during the rainy season. Such a huge amount of water has several consequences. From one side, the soil moisture of the terrain strongly increases and the vegetation can quickly grow up. These phenomena provoke an improvement of the backscattered field. From the other side, the soil roughness can decrease in wet terrains, causing a decrease of the backscattered field.
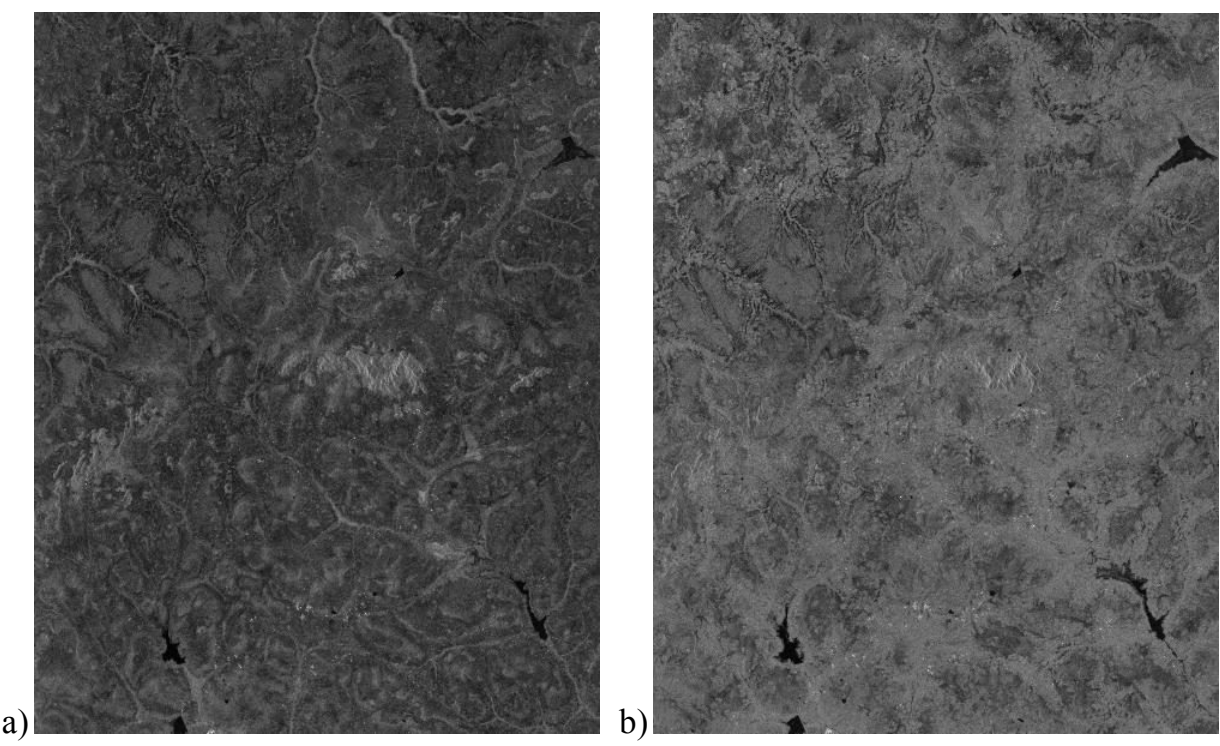

Figure 2. Example of stripmap Cosmo SkyMed images of the Ouahigouya area acquired on July, 14, 2010 (a) and August, 15,2010 (b). The original image resolution is $3 \mathrm{~m} \mathrm{x} 3 \mathrm{~m}$; the pixel spacing is $1.22 \mathrm{~m} \mathrm{x} 1.9 \mathrm{~m}$. The images are averaged by a $8 \times 8$ factor. A further average is due to the image compression.

In order to use the data for hydrological modeling, the development of methods for extracting quantitatively the physical information is crucial. A variety of details absolutely new for data acquired from satellites appears, and its characterization is still object of scientific discussion. The data carries physical information on the area topography, morphology and on physical parameters of interest in agriculture. In the next sections, we present the innovative models and techniques developed for extracting part of this information.

\section{THE MODELS}

\subsection{Hydrological models}

In the above presented context, land-use and water resource management choices have a prominent role in order to minimize the effects of the extreme climate conditions. Any management policy that could interfere with the local climate balance needs a hydrological assessment to calibrate and to forecast the future effects. Territorial management and hydrologic modeling studies are intrinsically related to the spatial processes of the hydrologic cycle-system. A 
multitude of ways to classify rainfall-runoff models from deterministic to stochastic models, from physically-based to empirical and conceptual models, and the most distinctive, from lumped models to distributed models, exists to reproduce the complex nature of hydrologic processes determined by a number of highly interconnected water, energy and vegetation processes at various spatial scales [4]. In this regard the deep knowledge of the problem requires an integrated approach of the geographical information. Significant efforts have been made in the linkage of geographic information systems (GIS) and hydrological-models [5] improving great bulk data management and rapid parameter estimation tools. The overriding need, in the choice of the proper hydrological model, has been the possibility of a widespread diffusion of the tools, developed in the framework of the present work, between local Burkinabè users. This consideration drove the choice to software freely available to the public. The SWAT (Soil and Water Assessment Tool) model, developed by the USDA (Agricultural Research Service at the Grassland, Soil and Water Research Laboratory in Temple, Texas, USA) has been selected for these applications, because it holds all the requested features. The code intends to be a model that provides a direct support to the management decisions. Many physical processes could be modeled by SWAT (weather, hydrology, stream routing, sedimentation, crop growth, nutrients, pesticide, etc.). In this work pollutant processes are not considered and only physical observables inherent to the hydrologic balance (water resources, flood, erosion) will be included. The land phase of the hydrologic processes, the driving force behind the movement of sediments, nutrients or pesticides, is simulated by the model based on the following water balance equation:

$$
\mathrm{SW}_{\mathrm{t}}=\mathrm{SW}_{\mathrm{o}}+\sum_{\mathrm{i}=1}^{\mathrm{t}}\left(\mathrm{R}_{\text {day }}-\mathrm{Q}_{\text {surf }}-\mathrm{E}_{\mathrm{a}}-\mathrm{W}_{\text {seep }}-\mathrm{Q}_{\mathrm{gw}}\right)
$$

where, $\mathrm{SW}_{\mathrm{t}}(\mathrm{mm})$ is the final soil water content, $\mathrm{SW}_{\mathrm{o}}$ is the initial soil water content, $\mathrm{t}$ is the time (days), $\mathrm{R}_{\text {day }}$ is the amount of precipitation, $\mathrm{Q}_{\text {surf }}$ is the amount of surface runoff, $\mathrm{E}_{\mathrm{a}}$ is the amount of evapotranspiration, $\mathrm{W}_{\text {seep }}$ is the amount of water entering the vadose zone from the soil profile, and $\mathrm{Q}_{\mathrm{gw}}$ is the amount of return flow on day $\mathrm{i}(\mathrm{mm})$.

For the ET parameter SWAT provides different methods assessment. The runoff evaluation is approached basing on curve number method and Green \& Ampt infiltration equation. Percolation is modeled with a layered storage routing technique combined with a crack flow model. Loadings of flow from the upland areas to the main channel are routed through the stream network of the watershed using a process similar to HYMO. The SWAT also implements a Modified Universal Soil Loss Equation (MUSLE) for simulating soil erosion. The complexity of the simulated physical processes requires a great number of data. The hydro-morphological basin analysis requires the knowledge of topographical information, soil moisture, land cover and land use and so on. Most of this data can efficiently be acquired via remote sensing instruments, with a huge economic saving.

\subsection{Remote sensing models}

The use of SAR images for hydrological applications is often limited by the difficulty of extracting physical information from the speckled data. In this paper we present innovative models for the retrieving of information from SAR images, based on efficient and innovative algorithms for the image interpretation. The results of the models are used as a starting point for the development of techniques of production of value added information. In particular, we provide the image interpreter the chance to use simple relations between the image characteristics and the geometric and dielectric parameters of the observed surfaces. The innovative character of the proposed method is the intensive use of fractal models. It requires the development of new models for the electromagnetic scattering from fractal surfaces [6]. In this paper we focus the attention mainly on two applications: the retrieval of topographic information via interferometric classical techniques and the classification of the images by means of innovative fractal tools [7]. The details on the techniques are provided along with the preliminary results in Section 4.

\subsection{Data integration}

The strong interdisciplinary character of the project drove to the need of integrating data coming from different sources. To this purpose, the Geographic Resources Analysis Support System (GRASS) open-source GIS was chosen. 


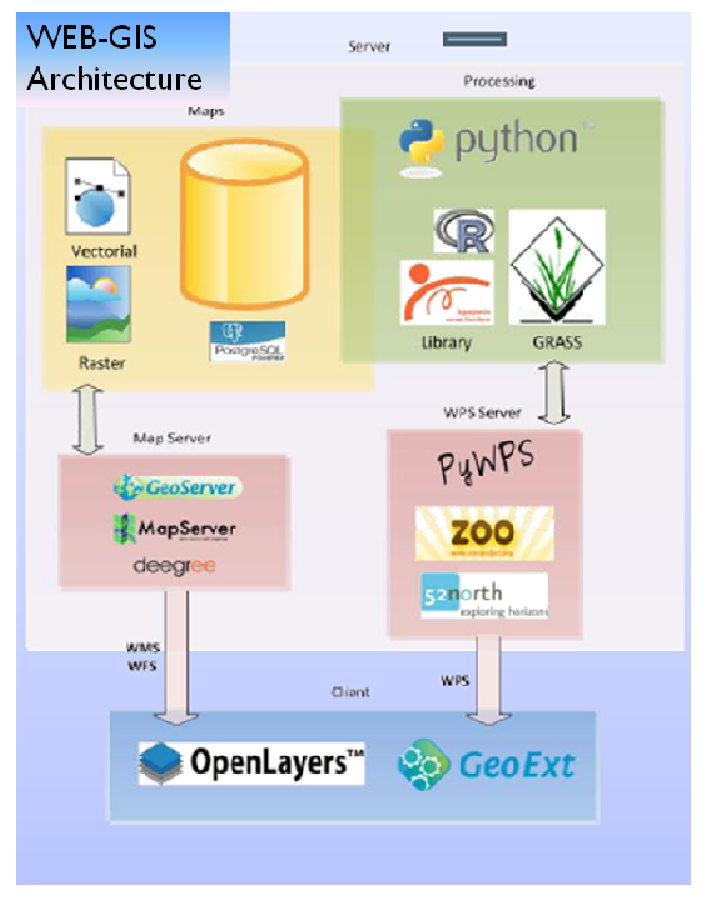

Figure 3. Web-GIS architecture.

We integrated data and models in the GIS, by adding new functions for fitting functional requirements, and producing new data with a surplus value. This process is dynamic and the data flow between the stakeholders is not fixed. So, while the analysis and development processes are in progress, new input data may become available bearing further information. Accordingly, an important requirement for the proposed instrument is the flexibility, because the solutions implemented have to be easily modifiable and the software development must be focused on easy code maintenance, intensive code reuse and incremental development of small-sized functions aimed to improve and ease the use of GRASS. GRASS allows to combine many maps and overlay them to display them as a stacked map. Many logic, spatial and arithmetic operations can be applied to multilayer maps, which is a hallmark of the GIS. All the above cited characteristics have to be available on the web, for guaranteeing an almost real-time data sharing. In Figure 3 we present the architecture that we choose for the web-GIS development and integration in Web-based instruments.

\section{PRELIMINARY RESULTS}

The above presented data carries a lot of information, whose extraction is not immediate for non-expert users. A complete processing chain is in phase of development, in order to retrieve as much information as possible. A first topological result of the study catchment shape is obtained by the processing of the first available high resolution SAR data. A Width Function Instantaneous Unit Hydrograph (WFIUH) model provided an optimization of the hydrologic characterization. In this Section we focus our attention on two examples: the topography extraction, which is performed via classical techniques, but that represent a strong value added for hydro-geological modeling; an innovative fractal technique for classifying SAR images.

\subsection{Topographic Information}

The configuration of the Cosmo-SkyMed constellation give the users the chance to acquire two images with the same orbit with a single day of time interaval. Therefore we used as input data a couple of SAR images acquired on the $12^{\text {th }}$ and the $13^{\text {th }}$ of June, 2010. Such a couple allowed the extraction of a digital elevation model (DEM) that was used to extract the main topographic information. Thank to the limited temporal shift and to the absence of significant vegetation 
at the beginning of the rainy season, the interferometric coherence, presented in Figure 4a is very high in most of the observed area. Some topographic characteristics can be already identified in the coherence information. The river streams can be noted as the dark, non coherent areas. Some villages, not easily visible in the intensity image, can be identified as bright spots.

a)

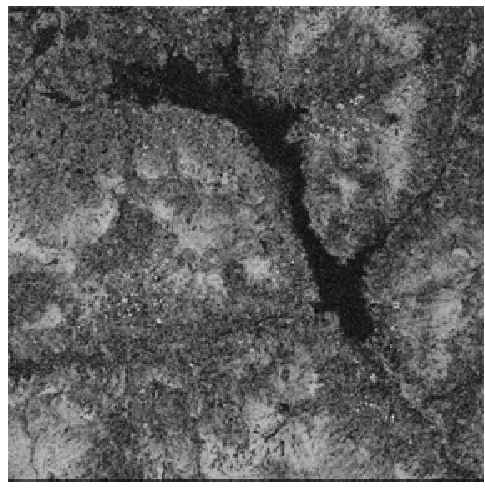

b)

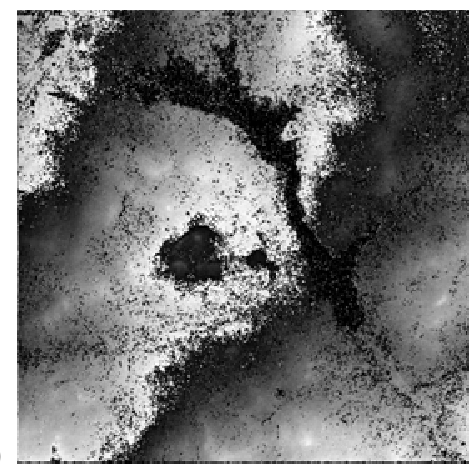

Figure 4. Interferometric coherence (a) and phase, after flat earth removal, (b) in the Tougou basin area.

Then, a classical interferometric chain was implemented via standard techniques. In Figure $4 \mathrm{~b}$ the interferometric phase (after flat Earth removal) is presented. Finally, the obtained georeferenced DEM is presented in Figure 5, resampled at $30 \mathrm{~m} \times 30 \mathrm{~m}$. A complete error budget analysis is still in the implementation phase. To this purpose, a measurement campaign is planned, so that we will compare the obtained results with in situ measurements, in order to assess the precision of the data.

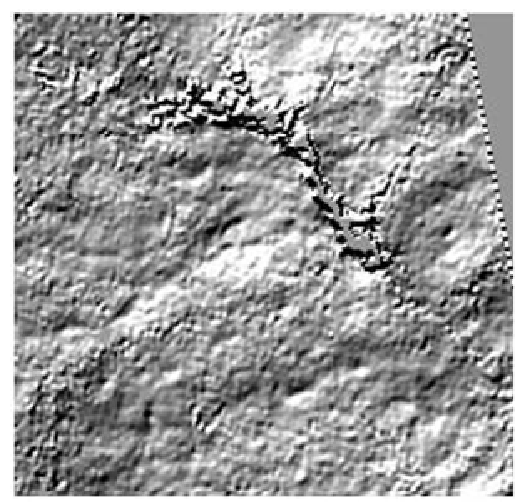

Figure 5. Sample of the obtained digital elevation model of the Tougou basin area.

\subsection{Innovative fractal classification detection study}

One of the innovative characteristics of the whole project is the implementation of fractal models in the data analysis. Fractal models are widely accepted as the most suitable geometrical models for accounting for the irregularities of natural surfaces. In particular, for the surface description we use the fractional Brownian motion (fBm), which is a continuous everywhere, differentiable nowhere process, described in terms of its increment pdf . A stochastic process $z(x, y)$ is an fBm surface if, for every $x, y, x^{\prime}, y^{\prime}$, it satisfies the following relation:

$$
\operatorname{Pr}\left\{z(x, y)-z\left(x^{\prime}, y^{\prime}\right)<\bar{\zeta}\right\}=\frac{1}{\sqrt{2 \pi} s \tau^{H}} \int_{-\infty}^{\bar{\zeta}} \exp \left(-\frac{\zeta^{2}}{2 s^{2} \tau^{2 H}}\right) d \zeta,
$$


where $\tau[\mathrm{m}]$ is the distance between the points $(x, y)$ and $\left(x^{\prime}, y^{\prime}\right)$, Pr stands for "probability" and the two parameters that control the fBm are the Hurst coefficient, $H$ (which is linked to the fractal dimension by the simple relation $D=3-H$ ) and the standard deviation of surface increments at unitary distance, $s\left[\mathrm{~m}^{1-H}\right]$.

It has been demonstrated [6] that an isotropic fBm exhibits a power law spectrum $S(k)$ :

$$
S(k)=S_{0} k^{-\alpha}
$$

wherein

$$
\begin{aligned}
& S_{0}=\frac{\pi H}{\cos (\pi H)} \frac{1}{\Gamma(1-2 H)} s^{2} \\
& \alpha=1+2 H=5-2 D
\end{aligned}
$$

$\Gamma$ being the Gamma function.

The expressions of the Power Spectral Densities (PSDs) of two cuts of the image in the range and azimuth directions respectively, have been evaluated by the authors in [7]. In particular, the spectrum of the image range cut, $S_{i}\left(k_{y}\right)$, in an appropriate range of sufficiently low spatial frequencies, presents a power law behavior whose exponent is related to the Hurst coefficient $H$ of the observed surface:

$$
S_{i}\left(k_{y}\right)=a_{1}^{2} s^{2} \Gamma(1+2 H) \operatorname{sen}(\pi H) \frac{1}{\left|k_{y}\right|^{2 H-1}}=S_{0}^{i}\left|k_{y}\right|^{-\alpha^{i}}
$$

wherein, $k_{y}$ is the wavenumber of the range cut of the image and $a_{1}$ depends on the assumed scattering model. In this paper the Small Perturbation Method (SPM) is used for modeling the electromagnetic scattering from the observed fractal surface [7]. In this case $a_{1}$ is a function of the fractal parameters of the imaged surface $(s, H)$ and of the sensor parameters (the electromagnetic wavenumber $\kappa$ and the sensor look angle $\theta_{0}$ ).

The relation in (6) is very useful for fractal parameter estimation purposes, because in log-log scale it represent a linear relation. Therefore, a linear regression allows to evaluate the slope, which is simply related with the fractal dimension [8]. Conversely, the retrieving of the second fractal parameter, the increment standard deviation $s$, starting from (6), deserves particular care. The SAR image power spectrum amplitude can depend not only on the amplitude coefficient of (6), but also on calibration parameters of the specific sensor that frequently cannot be completely controlled. For this reason we look for a relative value of $s$ that is retrieved considering the ratio of two SAR image power spectra. This ratio can be written as:

$$
\frac{s_{1}}{s_{2}}=\left(\frac{s_{i 1}\left(k_{y}\right)}{s_{i 2}\left(k_{y}\right)} \cdot \frac{1}{\mathrm{M}\left(\kappa, \theta_{0}, k_{y}, H_{1}, H_{2}\right)}\right)^{\frac{1}{4}}
$$

wherein $\mathrm{M}(\cdot)$ is a multiplicative function accounting for the different amplitude factors of the two spectra (see Eq. (6)). Note that $\kappa$ and $\theta_{0}$ are set for each sensor acquisition, while $H_{1}$ and $H_{2}$ can be estimated from the image through a linear regression as mentioned above. Hence, a reference patch can be defined on the image of interest and $s$ values of all the points of the image can be evaluated with respect to this reference $s$. Therefore, a sliding window can be used in order to obtain the maps of $D$ and of the relative $s$ of the observed surface.

In this paper we applied this algorithm to the case of the Tougou basin area in Burkina Faso, i.e. the area shown in Figure 6 . The obtained maps of the fractal dimension and of the relative incremental standard deviation $s\left[\mathrm{~m}^{H-H r e f}\right]$ are shown in Figure 7 and 8, respectively. In order to obtain these maps a sliding window of $51 \times 51$ pixels was used. The two maps provide complementary information on the area under survey and they could be combined to obtain classification maps and to investigate geological and hydrological properties of the observed soil. For example, it is known in the literature that the capillary wave spectrum of water basins presents a fractal behavior with $H=0.75$ and this is the value we retrieve in our maps on the zone relevant to the basin. 


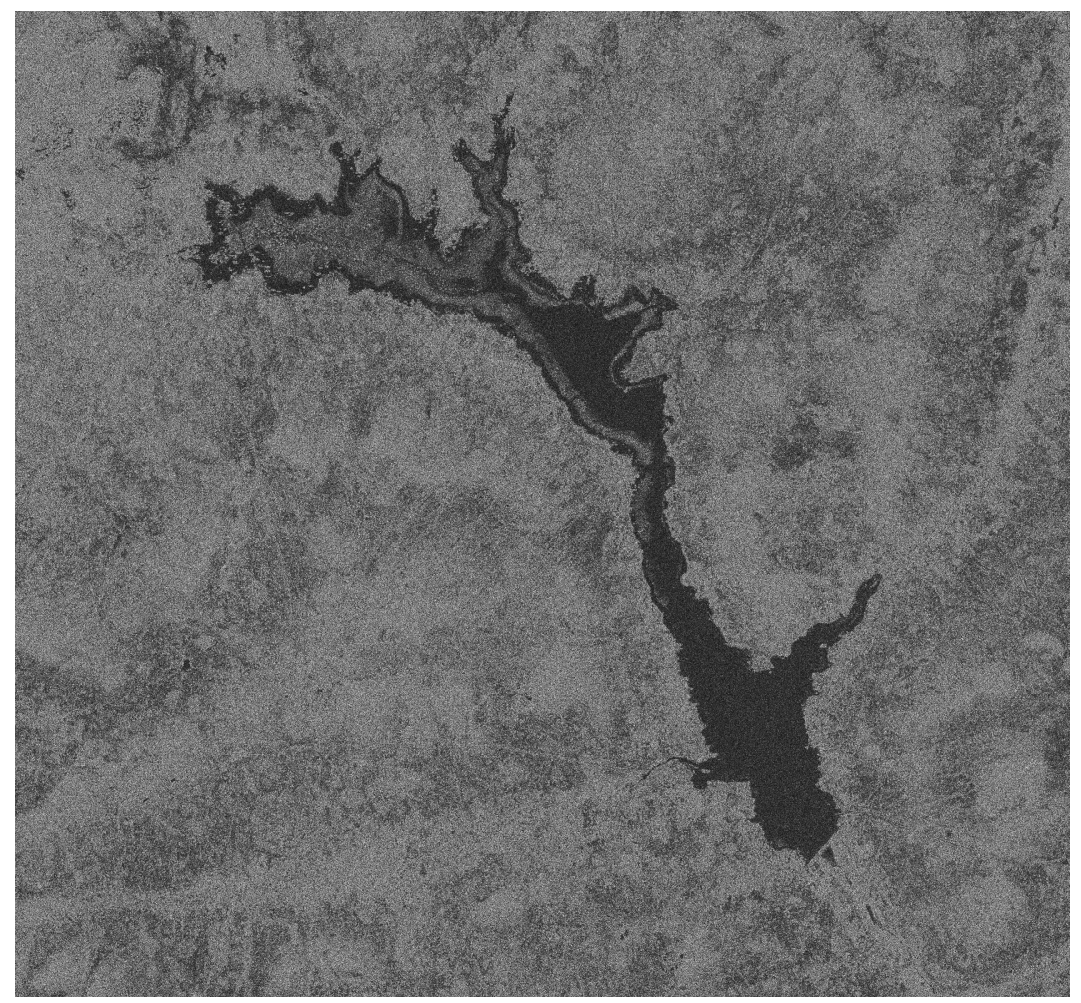

Figure 6. Amplitude image of the Tougou basin area.

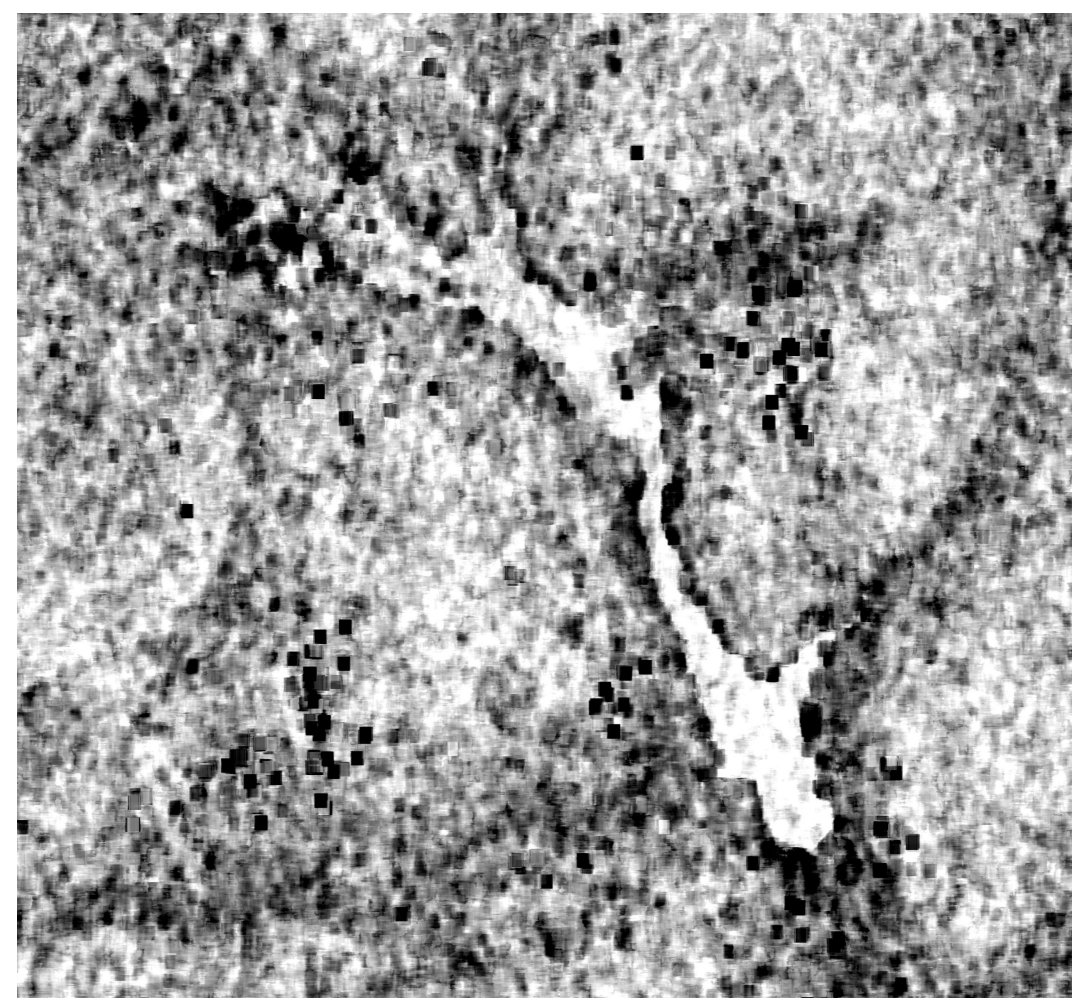

Figure 7. Fractal dimension map of the Tougou basin area. 


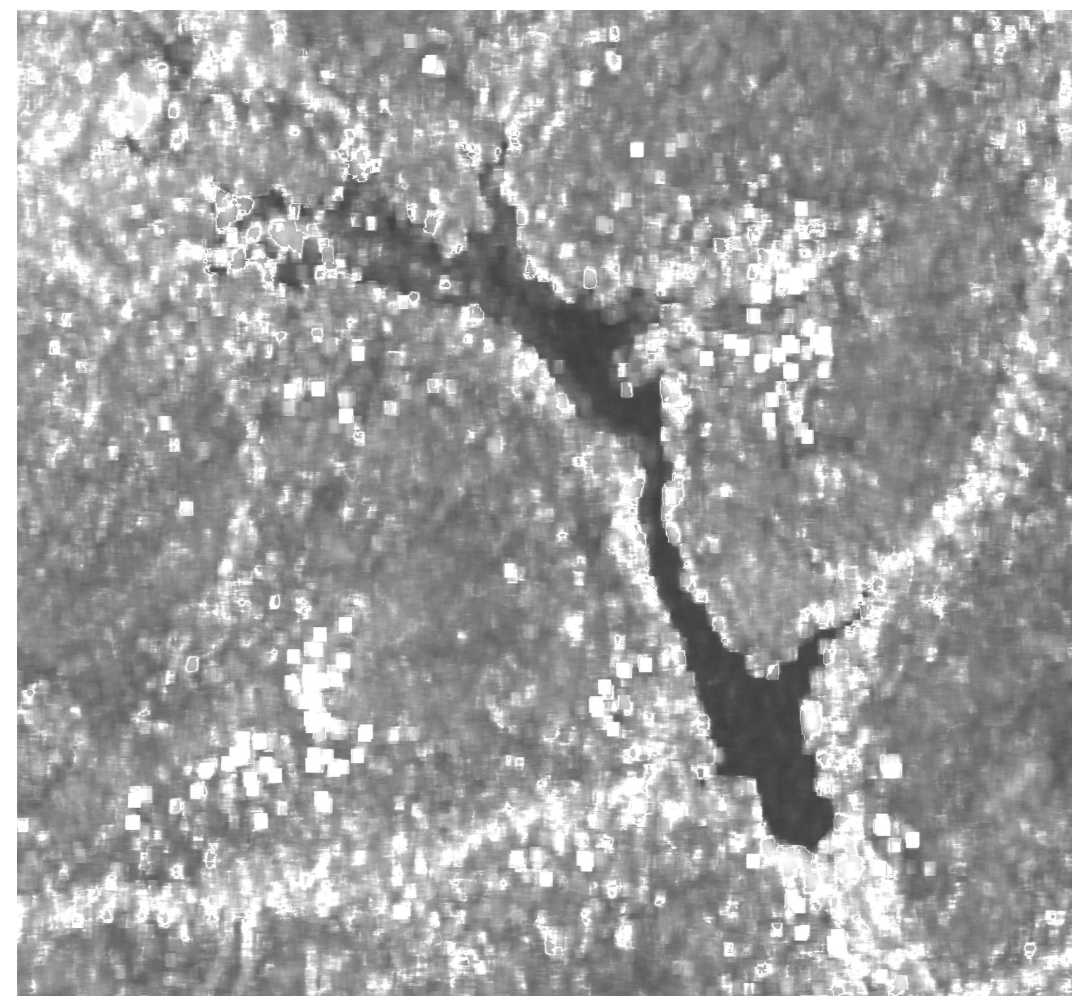

Figure 8. Map of the relative increment standard deviation $s$ relevant to the Tougou basin area.

\section{CONCLUSIONS}

The integration of heterogeneous data and models is a crucial factor for exploiting high resolution satellite data as a support for hydrologic modeling. In this paper we presented the rationale and the first results of a project devoted to extract physical information from high resolution COSMO-SkyMed data for calibrating hydrological models. A specific characteristic of the project is the use of open-source software for the hydrological and remote sensing model implementations, as well as for the geographic systems used for the data integration. Such a characteristic guarantees the availability of the final produced maps at almost no cost to the final users. In particular, in this paper we presented the preliminary results relative to the extraction of topographic information and an innovative classification fractal technique. The obtained results encourage to follow the idea that the new high resolution SAR data can provide an innovative and powerful instrument for monitoring developing countries at a limited cost with a benefit for the living condition of local people.

\section{ACKNOWLEDGMENTS}

This work has been supported in part by Agenzia Spaziale Italiana within COSMO/SkyMed AO, project ID 2218 and project ID 1200. 


\section{REFERENCES}

[1] Di Martino, G., A., Iodice, D., Riccio and Ruello, G., "A novel approach for disaster monitoring: fractal models and tools", IEEE Geosci. Remote Sens., 45, 1559-1570, (2007).

[2] Institut national de la statistique et de la démographie du Burkina Faso - INSD, "Recensement général de la population du Burkina Faso", (2006).

[3] Koussoubé, Y., "Hydrogéologie des séries sédimentaires de la dépression piézométrique du Gondo, (Bassin du Sourou) - Burkina Faso / Mali", Thèse de Doctorat, UPMC Sorbonne Universités, (2010).

[4] Beven, K. J. , "Distributed models", in Anderson, M. G. and Burt, T. P. Eds, (1985).

[5] Springer, D., "Towards integrating GIS Modelling and Software", 451-459, (2000).

[6] G. Franceschetti, and Riccio D., [Scattering, Natural Surfaces and Fractals]. Academic Press, Burlington (MA), USA, (2007).

[7] Di Martino, G., A., Iodice, D., Riccio, G., Ruello and Zinno I., "Fractal Based Filtering of SAR Images", Proc. IGARSS 2010, 2984-2987, (2010).

[8] Austin, T., A. W. England, G. H. Wakefield, "Special problems in the estimation of power-law spectra as applied to topographical modeling", IEEE Trans. Geosci. Remote Sens., (32), 928-939, (1994). 